\title{
Photorefractive Performance of Poly[methyl-3-(9-carbazolyl) propylsiloxane] Based Composites Sensitized with Poly(3-hexylthiophene) in a 0.2-1wt \% Range
}

\author{
Jin-Woo Oh* and Nakjoong Kim* \\ Department of Chemistry, Hanyang University, Seoul 133-791, Korea \\ *E-mail: daybreak0929@hanmail.net (JWO); kimnj@hanyang.ac.kr (NJK) \\ Received February 17, 2009, Accepted April 22, 2009
}

\begin{abstract}
In this work, we report on the characterization of six low- $T_{g}$ poly[methyl-3-(9-carbazolyl) propylsiloxane] based photorefractive (PR) composites sensitized with poly(3-hexylthiophene) (P3HT) in different concentrations, ranging from 0.2 to $1 \mathrm{wt} \%$. At $632.8 \mathrm{~nm}$, photoconductivity, space charge field, refractive index modulation, and grating buildup time were measured versus external electric field. The photoconductivity was strongly dependent on the visible light absorption and mobility. The magnitude of space charge field was affected by the conductivity contrast $\sigma_{\mathrm{ph}} /\left(\sigma_{\mathrm{ph}}+\sigma_{d}\right)$. The refractive index modulation increased with the magnitude of space charge field and the PR grating buildup speed increased with the photoconductivity.
\end{abstract}

Key Words: Poly(3-hexylthiophene), Space charge field, Refractive index modulation, PR grating buildup speed

\section{Introduction}

Since the first polymeric photorefractive (PR) composite $^{1}$ was reported in 1991, several polymeric composites with high PR performance were developed ${ }^{2-5}$ that compete with and in some aspects even surpass the performance level of the best currently known inorganic materials. ${ }^{6}$ Therefore, polymeric PR composites are today considered a highly promising class of new materials for optical applications. In polymeric PR composites, the photorefractivity depends on both the space charge field $\left(E_{s c}\right)$ and the reorientation of the chromophore by $E_{s c}$. A primary step in the formation of a space charge field is the creation of free electron-hole pairs via the absorption of a spatially modulated light intensity. In order to provide photosensitivity at the wavelengths of a commercially available low-cost He-Ne laser $(632.8 \mathrm{~nm})$, the photoconducting polymer matrix is often doped with a small concentration of an electron deficient molecule as a sensitizer. ${ }^{7}$ The sensitizer assists the PR material in generating photo-charge, which is governed by competition between the recombination of a charge carrier with its parent countercharge, which is termed geminate recombination, and electron-hole pair dissociation. To date, several classes of organic molecules have been used as sensitizers in organic PR materials. The choice of a sensi- tizer is often determined by the transport molecule (photoconductor), and the best performance is obtained by optimizing the charge-transfer properties between a chosen sensitizer and its parent photoconductor. ${ }^{8}$ The intermolecular interaction between the photoconductor and sensitizer leads to a new absorption band that does not appear in the spectrum of either component alone. Hence, spectral sensitivity can be achieved in the visible and the near infrared part of the spectrum using charge-transfer (CT) complexes. ${ }^{8,9}$ After generating free electron-hole pairs in the CT complex, the holes can migrate from one site to another with the aid of a strong electric field and become trapped in the dark region of the illumination pattern. ${ }^{7}$ As a result of the trapping process, the trapped charges form a spatially varying space charge field that can be translated into a variation in the refractive index through the Pockels effect using a nonlinear optical chromophore. ${ }^{7}$

In this work, we used a poly(3-hexylthiophene) (P3HT) as a sensitizer to achieve a high performance of polymeric PR composite. P3HT has been used recently in the fabrication of electronic devices, since it has good stability, reasonably high hole mobility, and a field effect mobility. ${ }^{10-12}$ By measurements of photoconductivities, space charge fields, refractive index modulations, and PR grating buildup times as a function of electric field, and sensitizer concentration, we have analyzed

Table 1. Composition, absorption coefficient $(\alpha)$, photoconductivity $\left(\sigma_{p h}\right)$, space charge field $\left(E_{s c}\right)$, refractive index modulation $(\Delta n)$, and grating buildup time $(\tau)$ of samples 1-6.

\begin{tabular}{ccccccccc}
\hline$\#$ & PSX-Cz & DB-IP-DC & P3HT & $\alpha\left(\mathrm{cm}^{-1}\right)^{a}$ & $\begin{array}{c}\sigma_{p h} \\
(\mathrm{pS} / \mathrm{cm})^{a, b}\end{array}$ & $\begin{array}{c}E_{s c} \\
(\mathrm{~V} / \mu \mathrm{m})^{a, b}\end{array}$ & $\begin{array}{c}\Delta n \\
\left(10^{-3}\right)^{a, b}\end{array}$ & $\begin{array}{c}\tau \\
(\mathrm{sec})^{a, b}\end{array}$ \\
\hline 1 & 69.8 & 30 & 0.2 & 109 & 0.362 & 4.61 & 1.73 & 5.40 \\
2 & 69.6 & 30 & 0.4 & 127 & 0.47 & 5.45 & 2.08 & 3.10 \\
3 & 69.4 & 30 & 0.5 & 136 & 0.527 & 5.50 & 2.20 & 2.15 \\
4 & 69.2 & 30 & 0.6 & 145 & 0.58 & 4.36 & 1.83 & 1.35 \\
5 & 69.2 & 30 & 0.8 & 163 & 0.815 & 3.52 & 1.30 & 0.75 \\
6 & 69.0 & 30 & 1.0 & 181 & 1.084 & 1.00 & 0.83 & 0.33 \\
\hline
\end{tabular}

${ }^{a}$ Measured at $\lambda=632.8 \mathrm{~nm} ;{ }^{b}$ Measured at $E_{0}=40 \mathrm{~V} / \mu \mathrm{m}$. 
(a)

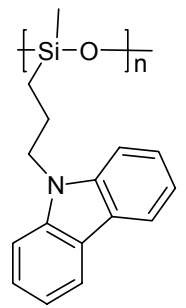

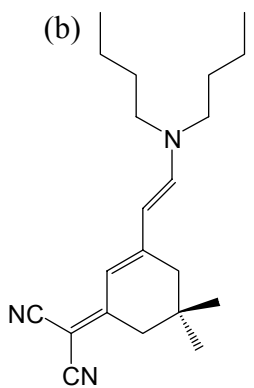

(c)

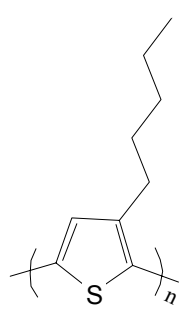

Figure 1. The chemical structure of the components in the PR composite: (a) PSX-Cz, (b) DB-IP-DC, and (c) P3HT.

some of the fundamental properties of P3HT-sensitized poly[methyl-3-(9-carbazoly) propylsiloxane] based composites. Increasing the $\mathrm{P} 3 \mathrm{HT}$ concentration changes the rate at which the mobile charges are generated in the material through increased absorption. ${ }^{7}$ As a result, a faster grating buildup would be expected with increasing sensitizer concentration.

\section{Experimental Section}

Materials and Sample Fabrications. In this work, six low $T_{g}$ photorefractive composites were prepared by doping the optically anisotropic chromophore, 2-[3-((E)-2-(dibutylamino)-1-ethenyl)-5,5-dimethyl-2-cyclohexenyliden] malononitrile (DB-IP-DC), into photoconducting polymer matrix, poly[methyl-3-(9-carbazolyl) propylsiloxane] (PSX-Cz) sensitized by poly(3-hexylthiophene) (P3HT). PSX-Cz and DBIP-DC were synthesized using previously described methods. ${ }^{13,14}$ P3HT obtained from Aldrich was used after purification. Figure 1 shows the chemical structures of the materials. The composition of polymeric composite was PSX-Cz : DBIP-DC : P3HT $=70-\mathrm{x}: 30: \mathrm{x}$ by wt $\%$. Table 1 shows the composition of our samples used in this study. For sample preparation, the mixtures (total $100 \mathrm{mg}$ ) were dissolved in 400 $\mathrm{mg}$ of dichloromethane and the solution was filtered through a $0.2 \mu \mathrm{m}$ membrane. The PR composites were cast on a patterned indium tin oxide (ITO) glass substrate, dried slowly for $12 \mathrm{~h}$ at ambient temperature, then heated in an oven to 90 ${ }^{\circ} \mathrm{C}$ for $24 \mathrm{~h}$ to completely remove the residual solvent. The composites were then softened on a hot plate at $100{ }^{\circ} \mathrm{C}$, and next sandwiched between ITO glasses with Teflon film spacer of $100 \mu \mathrm{m}$ to yield a film with a uniform thickness. ${ }^{13}$

Measurements. The conductivity $(\sigma)$ of the PR samples was measured at a wavelength of $632.8 \mathrm{~nm}$, using a simple dc current method. ${ }^{15}$ The current flowing through the sample was measured by the Keithley 6485 during illumination to be 20 $\mathrm{mW} / \mathrm{cm}^{2}$. The conductivity was calculated using the equation

$$
\sigma=\frac{J}{E}
$$

where $J$ is the current density, which is determined experimentally, and $E$ is the magnitude of the externally applied electric field.

The magnitude of the space charge field was measured

using the following method, reported in Ref. 16. In this method, the chromophore group, which had previously been aligned along the external electric field, was reoriented by the newly formed space charge field. A change in the birefringence was induced by the reorientation and was closely associated with the space charge field. Using numerical analysis based on the oriented gas model and the index ellipsoid method, the magnitude of the space charge field can be determined from the birefringence change.

The diffraction efficiency of photorefractive grating was determined by degenerated four-wave mixing (DFWM) experiments. Photorefractive grating was formed by the irradiation of two $s$-polarized beams with an intensity of $20 \mathrm{~mW} / \mathrm{cm}^{2}$ and a spot size of $6 \mathrm{~mm}$ in order to minimize the beam coupling between the writing beams, which causes the variation of photorefractive grating throughout the sample. Then the recorded photorefractive grating was read out by a $p$-polarized counter-propagating probe beam with an intensity of 0.06 $\mathrm{mW} / \mathrm{cm}^{2}$ and a spot size of $1.5 \mathrm{~mm}$. Two coherent laser beams with $\lambda=632.8 \mathrm{~nm}$ were irradiated on the composite in the tilted geometry at an incident angle of $\theta=30^{\circ}$ and $60^{\circ}$ with respect to the composite's normal axis. The magnitude of the diffraction efficiency $(\eta)$ was determined from the measured transmitted and diffracted intensities of the reading beam, ${ }^{14}$ using the relation

$$
\eta=I_{R, \text { diffracted }} /\left(I_{R, \text { diffracted }}+I_{R, \text { transmitted }}\right)
$$

The PR grating buildup times of the photorefractive composite were calculated by fitting the evolution of the growth of the diffraction signal. ${ }^{17}$

\section{Results and Discussion}

The absorption spectra of six composites are shown in Figure 2. All the photosensitizers were found to form a CT complex with PSX-Cz in the solid state. ${ }^{14}$ In the $550-800 \mathrm{~nm}$ wavelength range, the absorbance of composite increased with increasing the P3HT concentration. For sample 6 the absorbance was one and a half times more than in sample 1. In sample 1-6, where the equilibrium concentration of CT complex increases with the available amount of sensitizer, the absorption tails extend further into the long wavelength with increasing sensitizer concentration. The absorption coefficients $(\alpha)$ for samples 1-6 (at $632.8 \mathrm{~nm}$ of $100 \mu$ m thickness) were $109,127,136,145,163$, and $181 \mathrm{~cm}^{-1}$, respectively.

The photoconductivity $\left(\sigma_{p h}\right)$ is related to the number density $(p)$ of free charges produced by light absorption and the charge mobility $(\mu)$ by ${ }^{18}$

$$
\sigma_{p h}=p e \mu=\left(\frac{\varphi \alpha I_{q} \tau}{h v}\right) e \mu
$$

where $p$ is the density of mobile charge carriers (holes), $e$ is the fundamental electric charge, $\mu$ is the mobility of holes, $\varphi$ is photo-charge generation efficiency, $I_{q}$ is the optical intensity, $\tau$ is the life time of charge, $h v$ is the energy of photon. From 


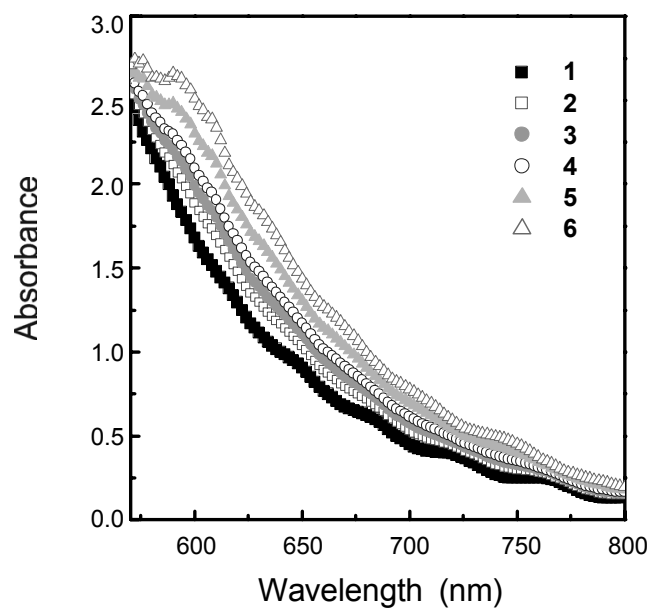

Figure 2. Visible spectrum of PR samples 1 (closed square), 2 (open square), 3 (closed circle), 4 (open circle), 5 (closed triangle), and 6 (open triangle).

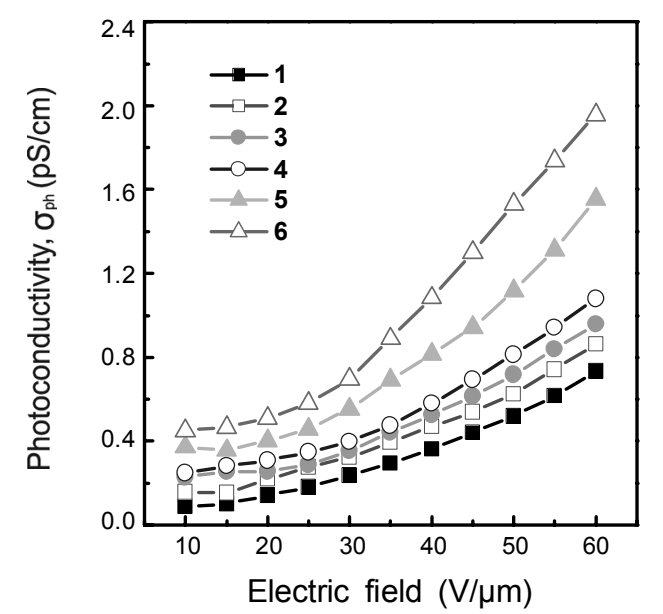

Figure 3. Field dependence of the photoconductivity measured in samples 1 (closed square), 2 (open square), 3 (closed circle), 4 (open circle), 5 (closed triangle), and 6 (open triangle). The line is a guide to the eye.

equation (3) we can confirm that the photoconductivity is governed by the charge generation and transport. ${ }^{19}$ All other things being equal, the inclusion of a photosensitizer results in an increase in $\sigma_{p h}$ and, because $\sigma_{p h}$ is an integral component of the PR effect, in an enhanced PR performance. The photoconductivity as a function of the applied electric field is presented in Figure 3. The photoconductivity was calculated as the difference between the total conductivity in the presence of light and dark conductivity in the absence of light. In Figure 3 the photoconductivity increased considerably with an increase in the electric field. This nonlinear dependence on the electric field is due to the electric field's dependencies on both the quantum efficiency of charge generation and the hole mobility. As shown in Figure 3, the photoconductivity increased with increasing the $\mathrm{P} 3 \mathrm{HT}$ concentration. The lager absorption and the faster mobility led to a significant increase in the photoconductivity. Figure 4 shows the dark conductivity as a func-

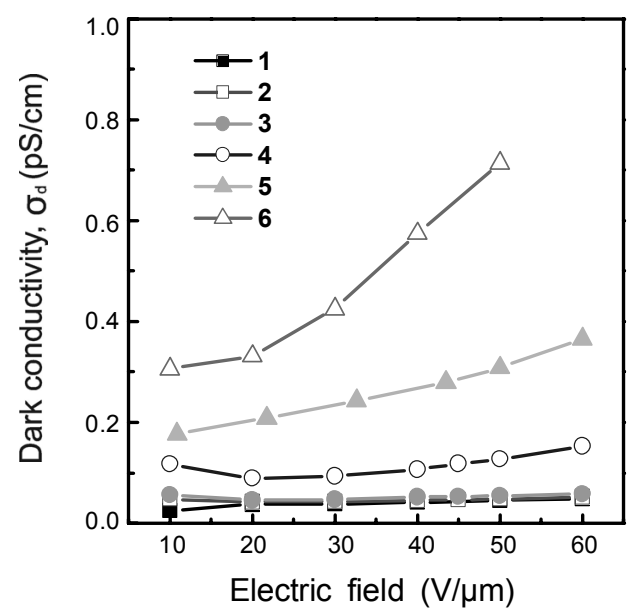

Figure 4. Field dependence of the dark conductivity measured in samples 1 (closed square), 2 (open square), 3 (closed circle), 4 (open circle), 5 (closed triangle), and 6 (open triangle). The line is a guide to the eye.

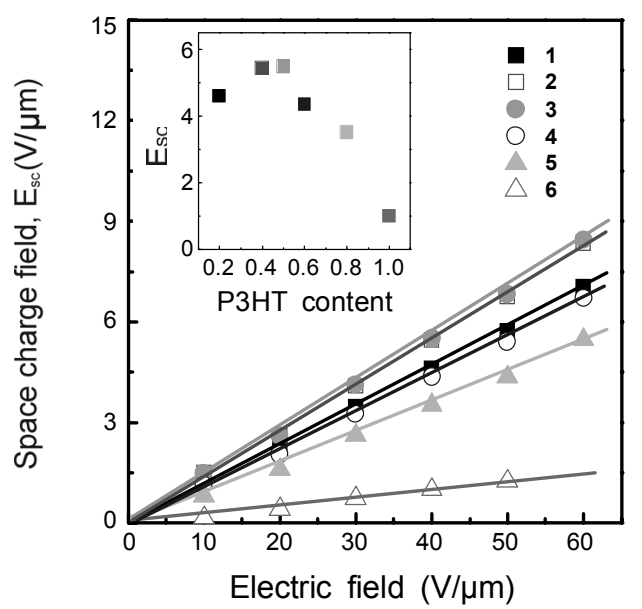

Figure 5. Field dependence of the space charge field measured in samples 1 (closed square), 2 (open square), 3 (closed circle), 4 (open circle), 5 (closed triangle), and 6 (open triangle). Inset shows P3HT concentration dependence of the space charge field in six samples. The line is a guide to the eye.

tion of the applied electric field. The dark conductivity $\left(\sigma_{d}\right)$ increased with increasing P3HT concentration. The above results will be explained in detail in the space charge field section.

We measured the space charge field of samples 1-6 as a function of the applied electric field. For clarity, Figure 5 shows the experimental data of the composites. $E_{s c}$ increased linearly with an increasing electric field. The electric field dependence of the $E_{s c}$ resulted from electric field-assisted separation of the charge from the electron-hole pair with a high energy distribution. ${ }^{14}$ As shown in Figure 5, the $E_{s c}$ of the six samples was in the following order: sample $6<$ sample $5<$ sample $4<$ sample $1<$ sample $2<$ sample 3 . The space charge field can qualitatively be attributed to the $\sigma_{\mathrm{ph}} /\left(\sigma_{\mathrm{ph}}+\sigma_{d}\right)$ ratio associated with PR sample. This so because the magnitude of the space charge field, $\left|E_{s c}\right|$, is correlated with the ratio $\sigma_{\mathrm{ph}} /\left(\sigma_{\mathrm{ph}}+\right.$ $\left.\sigma_{d}\right)$ as predicted by the equation ${ }^{20}$ 


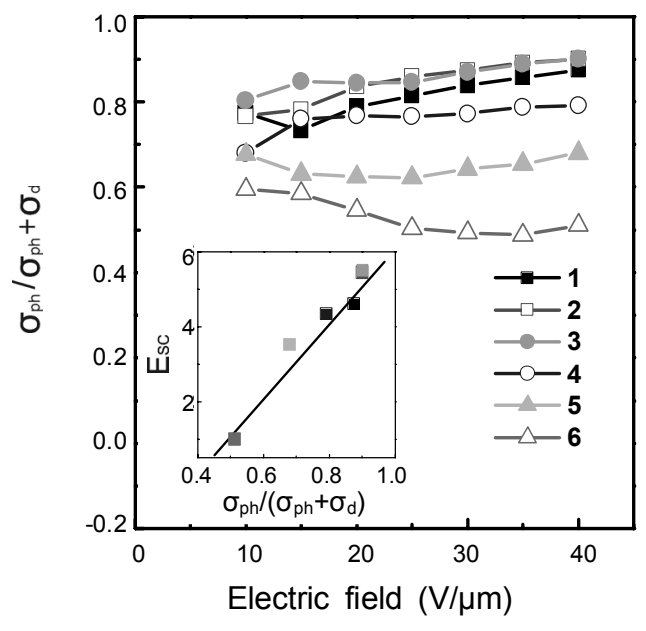

Figure 6. The ratio $\sigma_{\mathrm{ph}} /\left(\sigma_{\mathrm{ph}}+\sigma_{\mathrm{dark}}\right)$ as a function of field in samples 1 (closed square), 2 (open square), 3 (closed circle), 4 (open circle), 5 (closed triangle), and 6 (open triangle). Inset shows $\sigma_{\mathrm{ph}} /\left(\sigma_{\mathrm{ph}}+\right.$ $\left.\sigma_{\text {dark }}\right)$ dependence of the space charge field in six samples. The line is a guide to the eye.

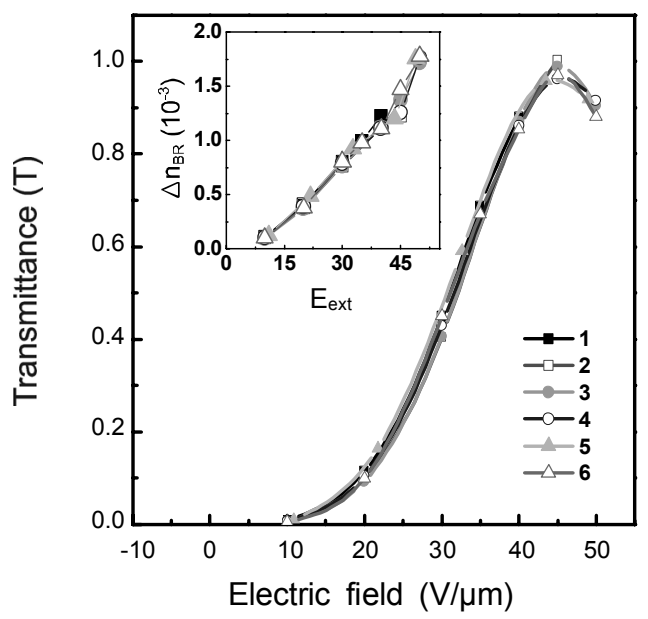

Figure 7. Field dependence of the steady-state transmittance measured in samples 1 (closed square), 2 (open square), 3 (closed circle), 4 (open circle), 5 (closed triangle), and 6 (open triangle). Inset shows field dependence of the birefringence measured in six samples. The line is a guide to the eye.

$$
\left|E_{s c}\right|=m \frac{\sigma_{p h}}{\sigma_{p h}+\sigma_{d}} \cdot \frac{E_{0} E_{q}}{\sqrt{E_{0}^{2}+E_{q}^{2}}}
$$

where $m$ is the modulation depth, and $E_{0}$ is the projection of applied electric fields along the grating wave vector. The saturation field $E_{q}=e \Lambda N_{T} /\left[2 \pi \varepsilon_{0} \varepsilon\right]$, where $\Lambda$ is the grating cons$\operatorname{tant}$, and $N_{T}$ is the PR trap density. Equation (4) assumes that the charge drift in the electric field dominates the diffusion of charges. ${ }^{19}$ Figure 6 shows the conductivity contrast $\sigma_{p h} /\left(\sigma_{p h}+\right.$ $\left.\sigma_{d}\right)$ of samples 1-6. The tendency of $\sigma_{p h} /\left(\sigma_{p h}+\sigma_{d}\right)$ has reserved at $3 \mathrm{wt} \%$ of $\mathrm{P} 3 \mathrm{HT}$ concentration where the space charge field has a maximum value. Below $3 \mathrm{wt} \%$, the $\sigma_{p h} /\left(\sigma_{p h}+\sigma_{d}\right)$ increases with increasing P3HT content due to the large absorption and the fast mobility. Above $3 \mathrm{wt} \%$, however, the $\sigma_{p h} /\left(\sigma_{p h}+\right.$ $\sigma_{d}$ ) decreases with the P3HT content. This is due to the increase

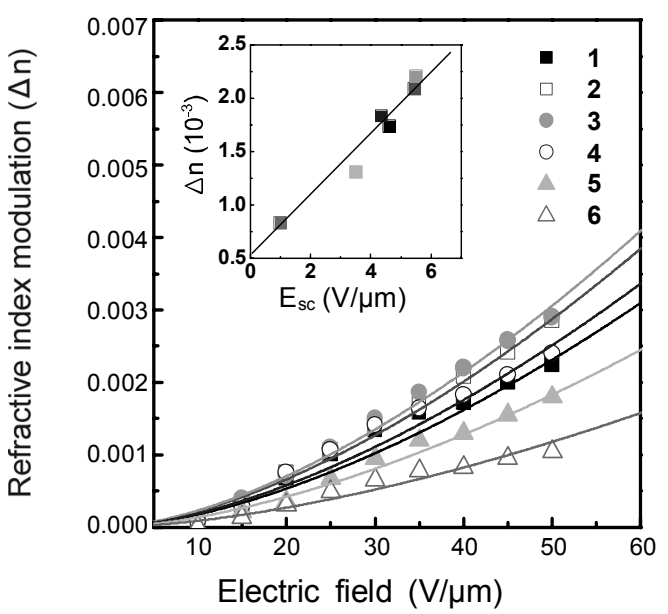

Figure 8. Field dependence of the refractive index modulation amplitude $\Delta n$ measured in samples 1 (closed square), 2 (open square), 3 (closed circle), 4 (open circle), 5 (closed triangle), and 6 (open triangle). Inset shows the correlation between the space charge field $\left(E_{s c}\right)$ and the refractive index modulation amplitude $(\Delta n)$ at $40 \mathrm{~V} / \mu \mathrm{m}$ in six samples. The fits are according to $n=a \cdot E^{b}$ (Ref. 14).

in hole detrapping by high dark conductivity. As shown in the inset in Figure 6, the space charge field increased linearly with the $\sigma_{p h} /\left(\sigma_{p h}+\sigma_{d}\right)$.

Differential scanning calorimetry experiments conducted on composites 1-6 yielded a broad range of glass transition temperatures between 20 and $30^{\circ} \mathrm{C}$. The ability of the chromophores to reorient in the composite was further confirmed using a transient ellipsometry technique. ${ }^{21}$ The transmittance $(T)$ as a function of the applied electric field for six samples is shown in Figure 7 . The birefringence $\left(\Delta n_{B R}\right)$ of samples was determined from the variation of the transmitted intensity through crossed polarizers upon the external electric field, as described by the following equation:

$$
T=\sin ^{2}\left(\frac{2 \pi}{\lambda} l \Delta n_{B R}\right)
$$

where $\lambda$ is wavelength, and $l$ is the distance of the light path. These results from the transmission ellipsometer experiments can be used to predict both the steady-state holographic contrast of the PR composite, and to quantify the rotational freedom of the chromophores within the sample. The birefringence of our samples strongly depended on the chromophore content. When describing chromophore alignment in the electric field, it is conventional to apply the oriented gas model, ${ }^{22}$ This model assumes be freely rotating, noninteracting molecules. In the low-field limit, the electric field-induced change in birefringence is found using the following equation. ${ }^{23,34}$

$$
\Delta n_{B R}=\frac{1}{2 \varepsilon_{0} n}\left(\frac{3}{45} N f_{\infty} \delta \alpha\left(\frac{\zeta}{k T}\right)^{2}+\frac{2}{3} N f_{0} f_{\infty}{ }^{2} \frac{\kappa \zeta}{5 k T}\right) E_{0}^{2}
$$

Here, $N$ is the chromophore concentration, $f_{0}, f_{\infty}$ are local field factors, $\delta \alpha$ is the polarizability anisotropy, $\kappa$ is the molecular first hyperpolarizability, and $\zeta$ is the dipole moment. Accor- 


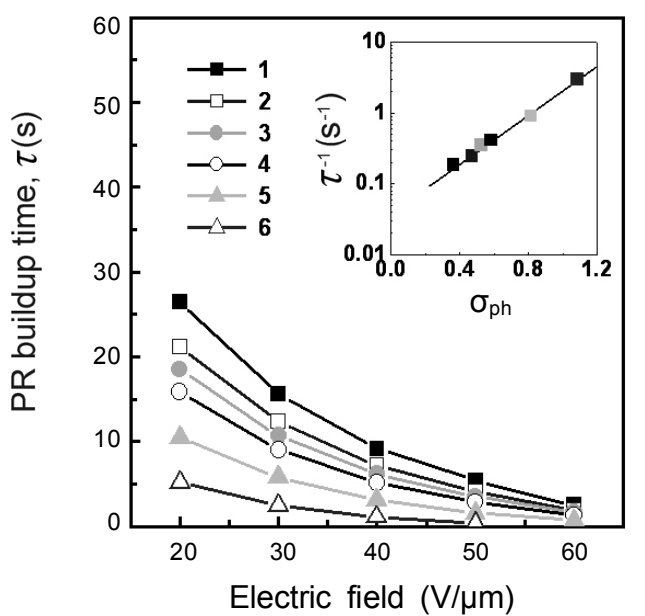

Figure 9. Field dependence of the grating buildup time $(\tau)$ in samples 1 (closed square), 2 (open square), 3 (closed circle), 4 (open circle), 5 (closed triangle), and 6 (open triangle). Inset shows the correlation between the photoconductivity $\left(\sigma_{p h}\right)$ and the PR grating buildup speed $(1 / \tau)$ at $40 \mathrm{~V} / \mu \mathrm{m}$ in six samples. The line is a guide to the eye.

ding to Equation (6), the steady-state electric-field induced $\Delta n_{B R}$ is dependent on the chromophore concentration. As shown in the inset of Figure 7, the $\Delta n_{B R}$ of six samples shows similar tendencies, since they have same content of chromophore.

The steady-state diffraction efficiencies of sample 1-6 were measured as a function of the applied electric field. The experiments were carried out at $T_{g}$ to take full advantage of the birefringence contribution to the index modulation amplitude. ${ }^{14}$ Figure 8 shows the refractive index modulation amplitudes, $\Delta n$, calculated using Kogelnik's expression for diffraction efficiency in thick transmission holograms, ${ }^{25}$

$$
\eta=\sin ^{2}\left[\frac{\pi d \Delta n \cos \left(\theta_{2}-\theta_{1}\right)}{\lambda \sqrt{\cos \theta_{1} \cos \theta_{2}}}\right]
$$

where $d$ is the composite thickness, and $\theta_{1}$ and $\theta_{2}$ are the internal angles of incidence of the two writing beams, respectively. The refractive index modulation of six samples was in the following order: sample $6<$ sample $5<$ sample $1<$ sample $4<$ sample $2<$ sample 3 . The index contrast of the photorefractive grating is given by the combination of the fieldinduced orientational birefringence factor and the space charge field as in equation (8),

$$
\Delta n \propto f(E)\left|E_{s c}(E)\right|
$$

where $f(E)$ is an orientational birefringence factor, which is a function of the internal angles of incidence and the coefficients defined in Equation (6). ${ }^{23}$ Since the orientational birefringence factors were fixed in all samples, the refractive index modulation is only dependent on the magnitude of the space charge field. The inset in Figure 8 shows the relation between the magnitude of space charge field and the refractive index modulation. The refractive index modulation directly increased with the space charge field which was dependent upon the ratio $\sigma_{\mathrm{ph}} /\left(\sigma_{\mathrm{ph}}+\sigma_{d}\right)$.

The field dependence of photorefractive grating buildup time was also analyzed. The PR grating buildup rate is very important for real applications such as real-imaging and realdata processing. ${ }^{26}$ The buildup time of the photorefractive composites was evaluated from the buildup of the beam intensity of the DFWM measurement. ${ }^{16}$ The time constants, $\tau_{1}$ and $\tau_{2}$, were calculated by fitting the evolution of the growth of the gain, $g(t)$, with the following biexponential function, ${ }^{27}$

$$
g(t)=a_{1}\left\{1-\exp \left(-t / \tau_{1}\right)\right\}+a_{2}\left\{1-\exp \left(-t / \tau_{2}\right)\right\}
$$

where $\tau_{1}$ and $\tau_{2}$ are the fast and slow time constants, respectively. Figure 9 shows the PR grating buildup times $\left(\tau_{l}\right)$ as a function of the external electric field. An elevated external electric field caused faster grating formation in all composites. This was expected since the charge mobility as well as the photo-charge generation efficiency both increased with the electric field. ${ }^{14}$ As shown in the inset in Figure 9, for samples 1-6 the PR grating buildup speed $\left(\tau^{-1}\right)$ increased linearly with the photoconductivity. Unlike the diffraction efficiency, which is dependent upon the ratio $\sigma_{\mathrm{ph}} /\left(\sigma_{\mathrm{ph}}+\sigma_{d}\right)$, the PR grating buildup speed is proportional to $\sigma_{p h}$, as given by the equation

$$
\tau^{-1}=\frac{\sigma_{\mathrm{ph}}}{\varepsilon}
$$

where $\varepsilon$ is the dielectric constant of the composite. ${ }^{28}$ Increasing the absorption coefficient with P3HT leads to a larger photoconductivity and a faster grating buildup through a faster photo-charge generation.

\section{Conclusions}

In this work, we investigated the steady-state and the dynamic properties of six PSX-Cz based composites, containing different amounts of P3HT. The photoconductivity, which was strongly dependent on the visible light absorption and mobility, increased with increasing the P3HT concentration. The magnitude of space charge field was affected by the conductivity contrast $\sigma_{p h} /\left(\sigma_{p h}+\sigma_{d}\right)$. The refractive index modulation increased with the magnitude of space charge field and the PR grating buildup speed increased with the photoconductivity. It would be very interesting to continue the investigation toward the improvement of the performance of PR polymers.

Acknowledgments. This work was supported by the National Research Foundation (NRF) grant funded by the Korea government (MEST) through the Active Polymer Center for Pattern Integration (No. R11-2007-050-00000-0) and by the Research fund of HYU (HYU-2008-T).

\section{References}

1. Ducharme, S.; Scott, J. C.; Twieg, R. J.; Moerner, W. E. Phys. Rev. Lett. 1991, 66, 1846. 
2. Cox, A. M.; Blackburn, R. D.; West, D. P.; King, T. A.; Wade, F. A.; Leigh, D. A. Appl. Phys. Lett. 1996, 68, 2081.

3. Lundquist, P. M.; Wortmann, R.; Geletneky, C.; Twieg, R. J.; Jurich, M.; Lee, V. Y.; Moylan, C. R.; Burland, D. M. Science 1996, 273, 1182.

4. Grunnet-Jepsen, A.; Thompson, C. L.; Twieg, R. J.; Moerner, W. E. Appl. Phys. Lett. 1997, 70, 1515.

5. Würthner, F.; Wortmann, R.; Matschiner, R.; Lakaszuk, K.; Meerholz, K.; De Nardin, Y.; Bittner, R.; Bräuchle, C.; Sens, R. Angew. Chem. Int. Ed. Engl. 1997, 36, 2765.

6. Bittner, R.; Bräuchle, C.; Meerholz, K. Appl. Opt. 1998, 37, 2843.

7. Steenwinckel, D. V.; Hendrickx, E.; Persoons, A. J. Chem. Phys. 2001, 114, 9557.

8. Hendrickx, E.; Kippelen, B.; Thayumanavan, S.; Mader, S. R.; Persoons, A.; Peyghambarian, N. J. Chem. Phys. 2000, 112, 9557.

9. Kim, Y. H.; Jung, S.-D.; Chung, M.-A.; Song, K. D.; Cho, D. W. Bull. Korean Chem. Soc. 2008, 29(5), 948.

10. Chiguvare, Z.: Dyakonov, V. Phys. Rev. B. 2004, 70, 234207.

11. Bao, W.; Dodabalapur, A.; Lobinger, A. J. Appl. Phys. Lett. 1996, 69, 4108.

12. Pang, I.; Boo, J.-H.; Sohn, H.; Kim, S.; Lee, J. Bull. Korean Chem. Soc. 2008, 29(7), 1349.

13. Kim, N.-J.; Chun, H.; Moon, I. K.; Joo, W.-J.; Kim, N. Bull. Korean Chem. Soc. 2002, 23, 571.

14. Oh, J.-W.; Moon, I. K.; Kim, N. J. Photochem. Photobio. A: Chem. 2009, 201, 222.
15. Schildkraut, J. S. J. Appl. Phys. 1991, 58, 340.

16. Joo, W.-J.; Kim, N. J.; Chun, H.; Moon, I. K.; Kim N. J. Appl. Phys. 2002, 91, 6471.

17. Oh, J.-W.; Choi, C.-S.; Kim, N. Mol. Cryst. Liq. Cryst. 2006, 477,133

18. Kipplen, B.; Meerholz, K.; Peyghambarian, N. In Nonlinear Optics of Organic Molecules and Polymers; Nalva, H. S.; Miyata, S., Eds.; CRC: Boca Raton, Fla: 1997; Chap. 8, p 465.

19. Oh, J.-W.; Joo, W.-J.; Moon, I. K.; Choi, C.-S.; Kim, N. J. Phys. Chem. B 2009, 113, 1592.

20. Winiarz, J. G. J. Phys. Chem. C 2007, 111, 1904.

21. Herlocker, J. A.; Ferrio, K. B.; Hendrickx, E.; Guenther, B. D.; Mery, S.; Kippelen, B.; Peyghambarian, N. Appl. Phys. Lett. 1999, 74, 2253.

22. Kuzyk, M. In Characterization Techniques and Tabulations for Organic Nonlinear Optical Materials; Kuzyk, M.; Drik, C., Eds.; Marcel Dekker, Inc.: New York, 1998; Vol. 60.

23. Moerner, W. E.; Grunnet-Jepsen, A.; Thompson, C. L. Annu. Rev. Mater. Sci. 1997, 27, 585.

24. Sandalphon; Kippelen, B.; Meerholz, K.; Peyghambarian, N. Appl. Opt. 1996, 35, 2346.

25. Kogelnik, H. Bell Syst. Tech. J. 1969, 48, 2909.

26. Oh, J.-W.; Lee, C.; Kim, N. J. Appl. Phys. 2008, 104, 073709.

27. Malliaras, G. G.; Krasnikov, V. V.; Bolink, H. J.; Hadziioannou, G. Appl. Phys. Lett. 1995, 66, 1038.

28. Oh, J.-W.; Kim, N. Chem. Phys. Lett. 2008, 460, 482. 